\title{
Androgen deprivation therapy prevents bladder cancer recurrence
}

Koji Izumi ${ }^{1}$, Masataka Taguri ${ }^{2}$, Hiroshi Miyamoto ${ }^{3}$, Yoshinori Hara ${ }^{4}$, Takeshi Kishida ${ }^{5}$, Kimio Chiba ${ }^{6}$, Tetsuo Murai ${ }^{7}$, Kotaro Hirai ${ }^{8}$, Kotaro Suzuki ${ }^{9}$, Kiyoshi Fujinami ${ }^{10}$, Teiichiro Ueki ${ }^{11}$, Koichi Udagawa ${ }^{12}$, Kazuo Kitami ${ }^{13}$, Masatoshi Moriyama ${ }^{14}$, Yasuhide Miyoshi ${ }^{15}$, Futoshi Tsuchiya ${ }^{16}$, Ichiro Ikeda ${ }^{17}$, Kazuki Kobayashi ${ }^{18}$, Maho Sato $^{2}$, Satoshi Morita ${ }^{19}$, Kazumi Noguchi ${ }^{15}$, Hiroji Uemura ${ }^{1}$

${ }^{1}$ Department of Urology, Yokohama City University Grasduate School of Medicine, Yokohama, Japan

${ }^{2}$ Department of Biostatistics and Epidemiology, Yokohama City University Medical Center, Yokohama, Japan

${ }^{3}$ Departments of Pathology and Urology, Johns Hopkins University School of Medicine, Baltimore, MD, USA

${ }^{4}$ Department of Urology, Odawara Municipal Hospital, Odawara, Japan

${ }^{5}$ Department of Urology, Kanagawa Cancer Center, Yokohama, Japan

${ }^{6}$ Department of Urology, Kawasaki Municipal Ida Hospital, Kawasaki, Japan

${ }^{7}$ Department of Urology, International Goodwill Hospital, Yokohama, Japan

${ }^{8}$ Department of Urology, Sagamihara National Hospital, Sagamihara, Japan

${ }^{9}$ Department of Urology, Saiseikai Yokohamashi Nanbu Hospital, Yokohama, Japan

${ }^{10}$ Department of Urology, Chigasaki Municipal Hospital, Chigasaki, Japan

${ }^{11}$ Department of Urology, Japanese Red Cross Hadano Hospital, Hadano, Japan

${ }^{12}$ Department of Urology, Hiratsuka Kyousai Hospital, Hiratsuka, Japan

${ }^{13}$ Department of Urology, Fujisawa City Hospital, Fujisawa, Japan

${ }^{14}$ Department of Urology, Yokohama Municipal Citizen's Hospital, Yokohama, Japan

${ }^{15}$ Department of Urology, Yokohama City University Medical Center, Yokohama, Japan

${ }^{16}$ Department of Urology, Yokohama City Minato Red Cross Hospital, Yokohama, Japan

${ }^{17}$ Department of Urology, Yokohama Minami Kyousai Hospital, Yokohama, Japan

${ }^{18}$ Department of Urology, Yokosuka Kyousai Hospital, Yokosuka, Japan

${ }^{19}$ Department of Biomedical Statistics and Bioinformatics, Kyoto University Graduate School of Medicine, Kyoto, Japan

Correspondence to:

Hiroji Uemura, e-mail: hu0428@med.yokohama-cu.ac.jp

Keywords: Bladder cancer, recurrence, androgen, androgen deprivation therapy

Received: November 05, $2014 \quad$ Accepted: December 03, $2014 \quad$ Published: December 24, 2014

\section{ABSTRACT}

Although accumulating preclinical evidence indicates the involvement of androgen receptor signals in bladder cancer (BC) development, its clinical relevance remains unclear. We aimed to evaluate the predictive role of androgen deprivation therapy (ADT) in BC recurrence in prostate cancer $(P C)$ patients.

We retrospectively reviewed 20,328 patients with PC diagnosed during 19912013 and identified $239(1.2 \%)$ men having primary BC. After excluding ineligible patients, 162 patients made up a final cohort.

With a median follow-up of 62 months, $38(50 \%)$ of 76 control patients without ADT experienced BC recurrence, while $19(22 \%)$ of 86 did in ADT group. Thus, patients having received ADT for their PC showed a significantly lower risk of BC recurrence (5-year actuarial recurrence-free survival: $76 \%$ v 40\%; $P<0.001$ ) and also had a significantly smaller number of recurrence episodes (5-year cumulative recurrence: 0.44 v 1.54; $P<0.001$ ), compared to the control patients. A multivariable analysis 


\section{revealed ADT as an independent prognosticator (hazard ratio, 0.29; $95 \%$ confidence interval, 0.17-0.49) for BC recurrence. This is the first clinical study showing that ADT significantly reduces the risk of $B C$ recurrence.}

\section{INTRODUCTION}

Urinary bladder cancer (BC) is the fourth and seventh most common malignancy in the US [1] and worldwide [2], respectively, in men. Most BCs are initially non-muscleinvasive and can be treated by transurethral resection (TUR). However, the recurrence rate of $\mathrm{BC}$ is quite high $(50 \%-70 \%)$ and progression to invasive state takes place occasionally [3]. Although bladder instillation therapy using anthracyclines or Bacillus Calmette-Guérin (BCG) is reported to be effective, the risk of recurrence even after the instillation treatment remains as high as $36 \%-51 \%$ [3]. In addition, due to the lifelong necessity to monitor the recurrence of $\mathrm{BC}$, the medical cost per $\mathrm{BC}$ patient from diagnosis to death is the highest among all cancer types [4].

One intriguing feature in $\mathrm{BC}$ is the three- to four-fold higher incidence in men than in women [1,2]. Although excessive exposure to industrial chemicals and cigarette smoke may have contributed to this male predilection, men remain at a substantially higher risk of $\mathrm{BC}$ than women even after controlling for these carcinogenic factors $[5,6]$. These findings prompted us to fathom the mechanism underlying such male predilection. In fact, we and others have found molecular evidence indicating, in animal models, that androgen receptor (AR) signals are involved in BC development [7-9]. It is therefore suggested that androgen-mediated activation of AR signaling would promote bladder tumorigenesis and cancer progression. There are basically two relevant states which may clearly explain the male predilection of BC. First, non-neoplastic urothelial and $\mathrm{BC}$ tissues in men express higher levels of $\mathrm{AR}$ and/or molecules related to its downstream pathway than those in women. Second, more simply, higher levels of circulating androgens in men would stimulate and maintain higher activation state of AR signaling in urothelial/ carcinoma cells. Examinations on the first hypothesis failed to demonstrate it to be the case [9-13]. Clinical studies have neither been successful to reveal the relationship between clinical course of patients or pathological state of BCs and AR level in BCs. On the other hand, the second hypothesis has not been examined so far in a well-controlled manner and remains to be an open question.

Prostate cancer (PC) is one of the most prevalent malignancies for men in developed countries [2]. As for its treatment, androgen deprivation therapy (ADT) stands as a critical treatment option [14]. It is used primarily for advanced PC in Europe and North America, while more widely used in Japan, even for some localized PC patients [15]. Interestingly, patients with PC are known to have a higher risk of coincidental $\mathrm{BC}$, reported to be $1.2-3.4 \%$ of
PC patients, than otherwise healthy men, probably due to shared molecular carcinogenic process between these two cancers [16].

Upon reviewing the background information, we have inclined to consider that circulating androgens would be responsible for AR-mediated gender difference in bladder tumorigenesis and cancer progression. If that is the case, we reasoned that ADT could influence the development of $\mathrm{BC}$ in $\mathrm{PC}$ patients. To test this hypothesis, we undertook a multicenter retrospective cohort study involving 20, 328 PC patients, including those having received ADT, and found surprisingly that ADT significantly reduced the risk of BC recurrence.

\section{RESULTS}

\section{Patient characteristics}

Table 1 shows the patient characteristics of the study cohort. The median follow-up for the entire cohort was 62 months (interquartile range, 19 to 95 months). Of the 162 patients, 86 were treated with ADT, and the remaining 76 did not receive ADT for their PC. The age of the patients was significantly higher in the ADT group than in the control group without ADT $(P=0.028)$. Among the reported clinicopathological factors of $\mathrm{BC}$ recurrence, i.e., tumor grade, stage, size, multiplicity and concomitant carcinoma in situ (CIS), the number of grade 2 tumors was significantly higher in the ADT group $(P=0.032)$. There was no significant difference in prophylactic bladder instillation therapy between the two groups. Regarding the features of PC, the prostate-specific antigen (PSA) level $(P=0.016)$ and tumor $\mathrm{T}$ stage $(P=0.047)$ were significantly higher, and brachytherapy was significantly less likely to have been performed $(P<0.001)$ in the ADT group compared to the control group.

\section{ADT and recurrence-free survival (RFS)}

We performed a Kaplan-Meier analysis coupled with a log-rank test to assess possible associations between ADT and $\mathrm{BC}$ recurrence. Overall, 19 (22\%) of the 86 patients with ADT versus $38(50 \%)$ of the 76 patients without ADT developed BC recurrence $(P<0.001)$. The 5 -year actuarial RFS rates for the ADT and control patients were $76 \%$ and $40 \%$, respectively. The ADT patients thus showed significantly improved RFS compared to the control patients without ADT $(P<0.001 ;$ Fig. 2$)$. To further explore the time-response relationship, we subdivided the patients into three groups according to ADT proportion 


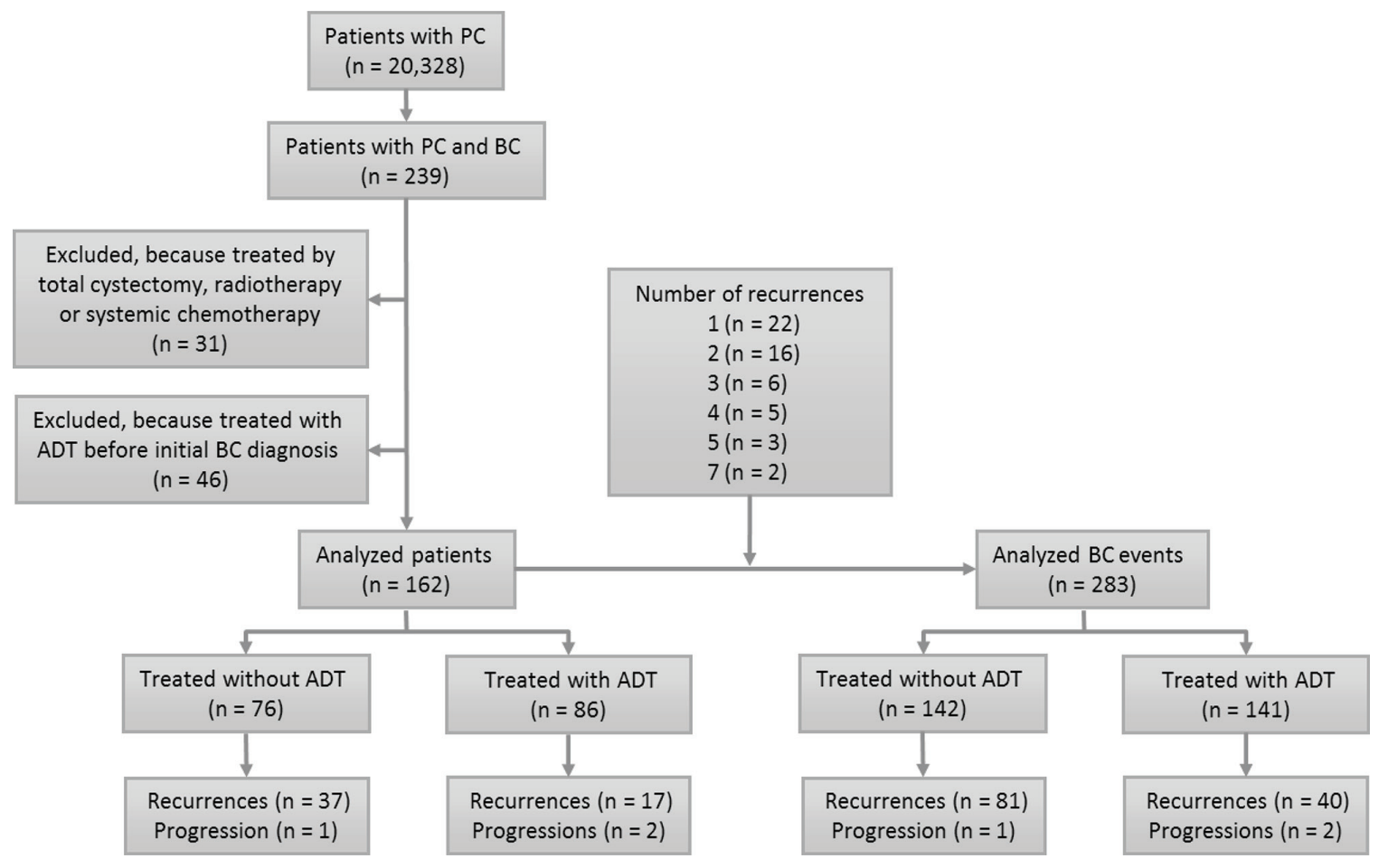

Figure 1: Flow of participants through study.

Table 1: Baseline Characteristics of the Patients with and without Androgen Deprivation Therapy (ADT)

\begin{tabular}{|c|c|c|c|c|}
\hline & Characteristics & Control, $n(\%)$ & ADT, $n(\%)$ & $P$ \\
\hline & No. of patients & $76(46.9)$ & $86(53.1)$ & \\
\hline & $\mathrm{Age}^{\mathrm{a}}, \mathrm{y}$ & $71.5(54-92)$ & $74.0(59-88)$ & 0.028 \\
\hline \multirow[t]{16}{*}{$\mathrm{BC}$} & Tumor grade & & & 0.032 \\
\hline & 1 & $26(35.6)$ & $16(20.5)$ & \\
\hline & 2 & $27(37.0)$ & $45(57.7)$ & \\
\hline & 3 & $20(27.4)$ & $17(21.8)$ & \\
\hline & Pathological T stage & & & 0.118 \\
\hline & $\mathrm{Ta}$ & $54(75.0)$ & $51(62.2)$ & \\
\hline & $\geq \mathrm{T} 1$ & $18(25.0)$ & $31(37.8)$ & \\
\hline & Tumor size & & & 1.000 \\
\hline & $<3 \mathrm{~cm}$ & $52(81.3)$ & $59(81.9)$ & \\
\hline & $\geq 3 \mathrm{~cm}$ & $12(18.8)$ & $13(18.1)$ & \\
\hline & Tumor number & & & 0.230 \\
\hline & Single & $33(52.4)$ & $30(41.7)$ & \\
\hline & Multiple & $30(47.6)$ & $42(58.3)$ & \\
\hline & Concomitant CIS & & & 0.241 \\
\hline & No & $60(89.6)$ & $64(82.1)$ & \\
\hline & Yes & $7(10.4)$ & 14 (17.9) & \\
\hline
\end{tabular}

(Continued) 


\begin{tabular}{|c|c|c|c|c|}
\hline & Characteristics & Control, $n(\%)$ & ADT, $n(\%)$ & $P$ \\
\hline & Instillation & & & 0.087 \\
\hline & No & $41(55.4)$ & $40(48.2)$ & \\
\hline & Anthracyclines & $21(28.4)$ & $17(20.5)$ & \\
\hline & BCG & $12(16.2)$ & $26(31.3)$ & \\
\hline \multirow[t]{25}{*}{$\mathrm{PC}$} & $\mathrm{PSA}^{\mathrm{b}}, \mathrm{ng} / \mathrm{ml}$ & $7.9(5.2-13.0)$ & $9.7(6.8-17.9)$ & 0.016 \\
\hline & Gleason score & & & 0.051 \\
\hline & $\leq 6$ & $30(43.5)$ & $21(25.0)$ & \\
\hline & 7 & $22(31.9)$ & $33(39.3)$ & \\
\hline & $\geq 8$ & $17(24.6)$ & $30(35.7)$ & \\
\hline & Clinical T stage & & & 0.047 \\
\hline & $\mathrm{T} 1$ & $47(64.4)$ & $42(50.6)$ & \\
\hline & $\mathrm{T} 2$ & $23(31.5)$ & $26(31.3)$ & \\
\hline & $\mathrm{T} 3$ & $3(4.1)$ & $14(16.9)$ & \\
\hline & $\mathrm{T} 4$ & $0(0.0)$ & $1(1.2)$ & \\
\hline & Clinical N stage & & & 0.500 \\
\hline & N0 & $72(100.0)$ & $82(97.6)$ & \\
\hline & N1 & $0(0.0)$ & $2(2.4)$ & \\
\hline & Clinical M stage & & & 0.123 \\
\hline & M0 & $75(100.0)$ & $81(95.3)$ & \\
\hline & M1 & $0(0.0)$ & $4(4.7)$ & \\
\hline & External beam radiotherapy & & & 1.000 \\
\hline & No & $61(87.1)$ & $67(87.0)$ & \\
\hline & Yes & $9(12.9)$ & $10(13.0)$ & \\
\hline & Brachytherapy & & & $<0.001$ \\
\hline & No & $47(70.1)$ & $71(93.4)$ & \\
\hline & Yes & $20(29.9)$ & $5(6.6)$ & \\
\hline & Radical prostatectomy & & & 0.227 \\
\hline & No & $59(88.1)$ & $72(94.7)$ & \\
\hline & Yes & $8(11.9)$ & $4(5.3)$ & \\
\hline
\end{tabular}

${ }^{a}$ Numbers are median (range). ${ }^{b}$ Numbers are median (interquartile range).

Abbreviations: ADT, androgen deprivation therapy; BC, bladder cancer; PC, prostate cancer; CIS, carcinoma in situ; BCG, Bacillus Calmette-Guérin; PSA, prostate-specific antigen.

that was defined as (treatment period with ADT)/(observed period for the first recurrence). The 5-year actuarial RFS rates based on ADT proportion $(0 \%, \leq 50 \%$, and $>50 \%)$ were $40 \%, 63 \%$, and $82 \%$, respectively, which were significantly different $(P<0.001$; Supplementary Fig. S1). These results indicate that ADT prevents $\mathrm{BC}$ recurrence in a time-dependent manner.

\section{ADT and the number of recurrence episodes}

Because BC patients often have multiple episodes of recurrence, we additionally analyzed the cumulative recurrence between the ADT and control groups. Among the 162 patients, 22 patients developed one recurrence, and 32 patients had more than one recurrence: two recurrences $(n=16)$, three $(n=6)$, four $(n=5)$, five $(n=3)$, and seven 


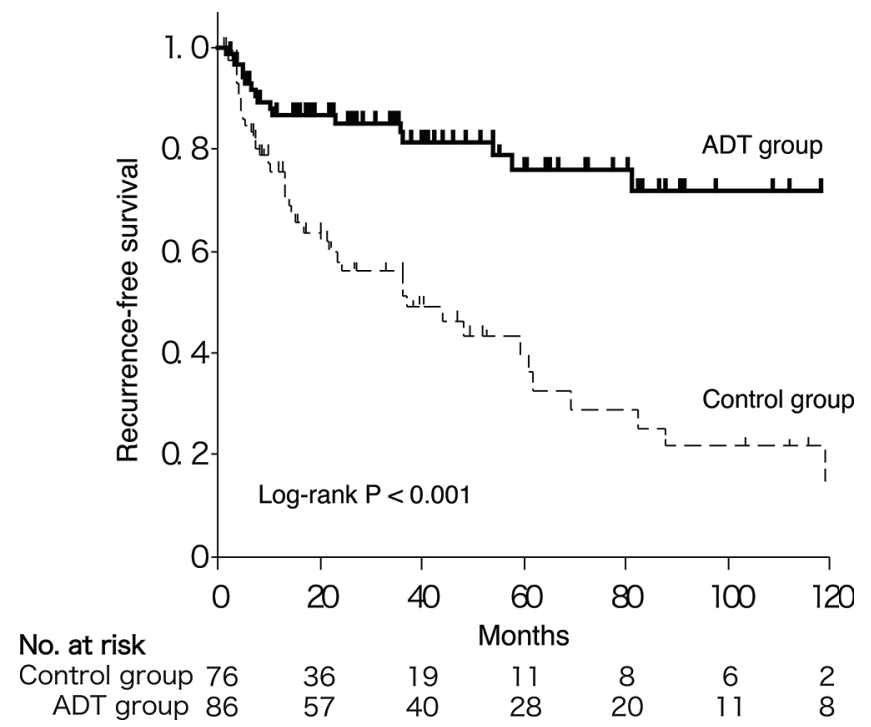

Figure 2: Kaplan-Meier curves for RFS in BC patients with versus without ADT.

$(n=2)$. Consequently, these patients had a total of $283 \mathrm{BC}$ events (initial BCs and recurrent BCs), including 141 in the ADT group and 142 in the control group (Fig. 1).

There were no statistically significant differences in the histopathologic features of BC between the ADT and control groups (Table 2). We then compared the cumulative recurrences of $\mathrm{BC}$ between the two groups. The number of 5 -year cumulative recurrence episodes was significantly smaller in the ADT group than in the control group (0.44 $v$ 1.54; $P<0.001$; Fig. 3). A Cox regression analysis demonstrated a lower risk of recurrence in the ADT group (hazard ratio (HR), 0.29; 95\% confidence interval (CI), 0.19 0.45; Table 3). Older age was also an independent predictor for recurrence (HR, 1.06; 95\% CI, 1.03-1.09; Table 3).

\section{Landmark analysis}

In an analysis of the effect of ADT on RFS, the length of the RFS would influence the chance of receiving ADT, thereby inducing a bias in favor of ADT. To minimize this bias, we conducted a landmark analysis with 12 months from the diagnosis as the landmark time point [17]. This analysis included patients who were at risk 12 months after diagnosis (the landmark time as determined a priori), and it compared the outcomes of those who had and had not received ADT in the prior 12 months. The patient backgrounds were not significantly different between the two groups, except for older age in the ADT group (Supplementary Table S1). Consistent with the results of all patients, the performance of ADT improved the RFS (Supplementary Fig. S2).

\section{DISCUSSION}

Molecular evidence has indicated that AR signals promote bladder carcinogenesis as well as cancer progression [9]. With the use of a chemical carcinogen N-butyl-N-(4- hydroxybutyl) nitrosamine (BBN) -induced BC model, castrated male and intact female mice were shown to have a lower incidence of $\mathrm{BC}$ compared to intact male mice. In addition, AR knockout in the entire body [7] or urothelium only [8] resulted in failure of BBN to induce BC. An AR degradation enhancer, ASC-J9, inhibited tumorigenesis in BBN-treated wild-type male mice [8]. Based on these data, androgen-mediated AR signaling in urothelial cells has been suggested to be a therapeutic target in $\mathrm{BC}$. In the present study, we showed, for the first time, clinical evidence implying that $\mathrm{ADT}$ prevents $\mathrm{BC}$ recurrence.

Incidence of $\mathrm{BC}$ in 20,328 PC patients in the current study was $1.2 \%$, which is consistent with previous findings [16]. Since we set the recurrence of BC as a primary endpoint instead of the incidence of $\mathrm{BC}$, we excluded patients who had already received ADT before initial BC diagnosis in order to circumvent a possible ADT effect on the characteristics of the initial BC. We were unable to assess the effect of ADT on BC progression because only three patients in our cohort developed muscle invasion. Of the 31 patients who had been treated by radical cystectomy, radiotherapy, or systemic chemotherapy, and were excluded from the analysis, eight developed metastasis or local invasion, which resulted in cancer-specific mortality. The effect of ADT on cancer-specific survival among these patients remains unclear because of the relatively small sample size and complicated background. To further investigate the effect of ADT on BC progression, prospective trials involving patients undergoing radical cystectomy are required, as Gakis et al. have suggested [18].

In our cohort, men who received ADT were older and had higher PSA/stage PC than those without ADT, probably because ADT was more likely used for older patients with advanced PC. The patients who underwent brachytherapy had lower Gleason scores and, as expected, were less likely treated with ADT. Although the reason why the ADT group had more grade $2 \mathrm{BCs}$ than the control group is unclear, 
Table 2: Clinicopathological Features of All BC Events with and without Androgen Deprivation Therapy (ADT)

\begin{tabular}{|l|l|l|l|}
\hline Characteristics & Control, $\boldsymbol{n}$ (\%) & ADT, $\boldsymbol{n}(\%)$ & \\
\hline No. of events & $142(50.2)$ & $141(49.8)$ & 0.011 \\
\hline Age ${ }^{a}$ y & $74.0(54-93)$ & $76.0(59-90)$ & 1.000 \\
\hline Tumor grade & & & \\
\hline 1 & $44(32.8)$ & $30(23.4)$ & \\
\hline 2 & $61(45.5)$ & $72(56.3)$ & \\
\hline 3 & $29(21.6)$ & $26(20.3)$ & 0.223 \\
\hline Pathological T stage & & & \\
\hline Ta & $102(75.6)$ & $92(68.1)$ & \\
\hline$\geq$ T1 & $33(24.4)$ & $43(31.9)$ & 0.442 \\
\hline Tumor size & & & \\
\hline$<3$ cm & $106(89.1)$ & $101(85.6)$ & \\
\hline$\geq 3$ cm & $13(10.9)$ & $17(14.4)$ & 0.697 \\
\hline Tumor number & & & \\
\hline Single & $55(46.2)$ & $52(43.3)$ & \\
\hline Multiple & $64(53.8)$ & $68(56.7)$ & \\
\hline Concomitant CIS & & & 0.861 \\
\hline No & $108(85.0)$ & $110(85.9)$ & \\
\hline Yes & $19(15.0)$ & $18(14.1)$ & \\
\hline Instillation & & $68(50.0)$ & \\
\hline No & $73(53.3)$ & $32(23.5)$ & \\
\hline Anthracyclines & $20(14.6)$ & \\
\hline BCG & & & \\
\hline
\end{tabular}

${ }^{a}$ Numbers are median (range).

Abbreviations: ADT, androgen deprivation therapy; CIS, carcinoma in situ; BCG, Bacillus Calmette-Guérin.

$\mathrm{ADT}$ prevents $\mathrm{BC}$ recurrence even if cases are separately analyzed in each grade. In addition, among all of these clinicopathologic factors, only patient age was an independent prognosticator for $\mathrm{BC}$ recurrence in our multivariable analysis. The result of the age as one of the independent prognosticators for RFS in this study is consistent with the epidemiological finding indicating that elderly men have a higher incidence of $\mathrm{BC}$. It has been reported that subsequent $\mathrm{BC}$ incidences are not different between the $\mathrm{PC}$ patients who underwent radical prostatectomy and radiation therapy [19]. Local treatments for PC (radical prostatectomy, external beam radiation therapy, and brachytherapy) had no or little influence on $\mathrm{BC}$ recurrence (Table 3).

We found that ADT dramatically prevented the first $\mathrm{BC}$ recurrence (Fig. 2). In addition, the ADT proportion correlated with the RFS (Supplementary Fig. S1), suggesting that the preventive effect of ADT on BC recurrence was time-dependent. Moreover, the cumulative recurrence analysis indicated that $\mathrm{BC}$ recurrences were again strongly suppressed ( $\mathrm{HR}, 0.29 ; 95 \% \mathrm{CI}, 0.17$ 0.49 ; Table 3) as long as the patients received ADT. Our landmark analysis excluded the possible bias that patients with longer RFS might have more likely received ADT.

We also observed that BCG instillation therapy tended to be more frequent in the ADT group, which might have affected the favorable RFS. However, our multivariable analysis revealed ADT, but not BCG instillation therapy, as an independent prognostic factor for $\mathrm{BC}$ recurrence (Table 3). These unexpected results further confirmed the preventive role of ADT in BC recurrence. Since gonadotropin-releasing hormone agonists were used as ADT forms in more than $90 \%$ of our patients, it remained unclear whether anti-androgen alone sufficiently prevented $\mathrm{BC}$ recurrence. Assessments of AR expression and androgen levels are needed to determine whether the preventive effect of $\mathrm{ADT}$ on $\mathrm{BC}$ recurrence is mediated by androgen-mediated 
AR signals or other possible pathways, such as estrogen receptor signals [20, 21].

Accumulating evidence suggests the involvement of various molecules that modulate $\mathrm{AR}$ activity and/ or are regulated by AR signals in $\mathrm{BC}$ development. These included epidermal growth factor receptor/ ERBB2 [12, 22, 23], Wnt/ $\beta$-catenin [24, 25], p53 [8], UDP-glucuronosyltransferase-1A [26, 27], and CD24 [28]. The results of the present study strongly encourage further investigations of these downstream pathways of AR signals with the goal of developing a new therapeutic approach (in addition to standard forms of ADT) for BC.

The recurrence rate in $\mathrm{BC}$ patients undergoing bladder instillation therapy using an anthracycline or BCG remains high [3]. New preventive approaches for BC recurrence are thus urgently required. According to our cumulative recurrence analysis, roughly five person-years of ADT prevents one $\mathrm{BC}$ recurrence (Fig. 3). Because ADT has been widely used for PC and is generally welltolerated, the clinical application of ADT to BC patients is feasible. We are planning to conduct a randomized clinical trial to further assess the efficacy of ADT in BC recurrence.

The limitation of the present study is that it was a multicenter retrospective study design. In the present study, reported prognosticators, such as tumor grade, stage, size, multiplicity and concomitant CIS [29], as well as prophylactic instillation therapy with BCG or anthracyclines, were not significantly associated with $\mathrm{BC}$ recurrence, probably due to the relatively small sample size.

In conclusion, in men with double primary cancers of the bladder and prostate, ADT for PC resulted in a significant decrease in the risk of $\mathrm{BC}$ recurrence. These results strongly suggest the involvement of androgenAR signals in $\mathrm{BC}$ recurrence, as well as the efficacy and feasibility of ADT for patients with BC.

\section{METHODS}

\section{Patients}

Institutional review board approval was obtained to conduct this study. We retrospectively identified a total of 239 patients with primary BC, from 20,328 men with PC, between January 1991 and May 2013 at Yokohama City University and 15 affiliated hospitals. All BCs were graded in accord with the 1973 WHO classification systems for urothelial neoplasms [30], at each institution. Thirty-one patients were excluded because they had undergone radical cystectomy $(n=27)$, radiotherapy $(n=2)$, chemotherapy $(n=1)$, or chemoradiotherapy $(n$ $=1$ ) as an initial treatment for BC. Additional 46 patients who had already been treated with ADT before the initial diagnosis of $\mathrm{BC}$ were also excluded. The remaining 162 patients formed the study cohort for analysis (Fig. 1).

In general, the patients were followed every three months with cystoscopy for two years from the time of the initial treatment of their $\mathrm{BC}$ and every six months thereafter. All BC recurrences were pathologically confirmed in TUR specimens. Three patients who progressed to muscle-invasive $\mathrm{BC}$ were determined as recurrence and censored. The outcome measures included RFS and cumulative recurrence of BC. The ADT group included patients treated with ADT for PC at any time from the diagnosis of $\mathrm{BC}$ to recurrence or censoring.

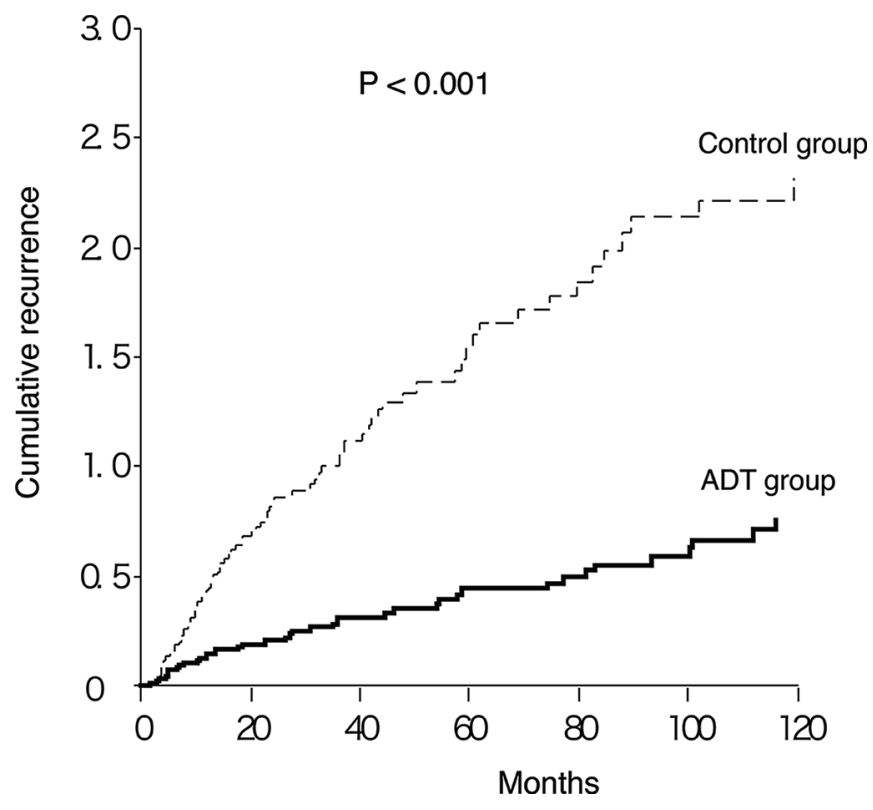

Figure 3: Cumulative recurrence of $\mathrm{BC}$ in patients with versus without ADT. 
Table 3: Univariable and Multivariable Analyses for Cumulative Recurrence

\begin{tabular}{|c|c|c|c|c|c|}
\hline & \multirow[b]{2}{*}{ Variables } & \multicolumn{2}{|c|}{ Univariable analysis } & \multicolumn{2}{|c|}{ Multivariable analysis } \\
\hline & & HR (95\%CI) & $\boldsymbol{P}$ & HR $(95 \%$ CI $)$ & $\boldsymbol{P}$ \\
\hline & ADT (yes vs. no) & $0.29(0.17-0.49)$ & $<0.001$ & $0.29(0.19-0.45)$ & $<0.001$ \\
\hline & Age, continuous value & $1.04(1.01-1.07)$ & 0.005 & $1.06(1.03-1.09)$ & $<0.001$ \\
\hline \multirow[t]{8}{*}{$\mathrm{BC}$} & Tumor grade (2 vs. 1$)$ & $1.38(0.80-2.38)$ & 0.247 & $1.19(0.72-1.96)$ & 0.507 \\
\hline & Tumor grade (3 vs. 1 ) & $1.10(0.52-2.31)$ & 0.805 & $0.66(0.31-1.41)$ & 0.282 \\
\hline & pT stage $(\geq \mathrm{T} 1$ vs. $\leq \mathrm{Ta})$ & $0.82(0.51-1.33)$ & 0.422 & $1.16(0.61-2.18)$ & 0.656 \\
\hline & Tumor size $(\geq 3 \mathrm{~cm}$ vs. $<3 \mathrm{~cm})$ & $1.02(0.51-2.06)$ & 0.952 & $1.04(0.54-2.03)$ & 0.903 \\
\hline & Tumor number (Multiple vs. Single) & $1.02(0.65-1.60)$ & 0.933 & $1.08(0.72-1.63)$ & 0.712 \\
\hline & CIS (yes vs. no) & $0.91(0.44-1.91)$ & 0.811 & $1.44(0.67-3.09)$ & 0.350 \\
\hline & Anthracylines (yes vs. no) & $0.87(0.54-1.41)$ & 0.580 & $0.69(0.43-1.10)$ & 0.117 \\
\hline & BCG (yes vs. no) & $0.47(0.26-0.84)$ & 0.011 & $0.59(0.30-1.18)$ & 0.137 \\
\hline \multirow[t]{10}{*}{$\mathrm{PC}$} & Gleason Score $(\geq 7$ vs. $\leq 6)$ & $0.83(0.43-1.61)$ & 0.582 & & \\
\hline & Gleason Score $(\geq 8$ vs. $\leq 6)$ & $1.09(0.56-2.13)$ & 0.793 & & \\
\hline & Gleason Score ( $\geq 8$ vs. $\leq 7)$ & $1.21(0.68-2.13)$ & 0.521 & & \\
\hline & PSA, continuous value & $1.05(0.60-1.84)$ & 0.865 & & \\
\hline & cT stage $(\geq \mathrm{T} 3$ vs. $\leq \mathrm{T} 2)$ & $1.36(0.66-2.81)$ & 0.402 & & \\
\hline & cN stage (N1 vs. N0) & $1.29(0.12-13.37)$ & 0.832 & & \\
\hline & cM stage (M1 vs. M0) & $1.75(0.34-8.98)$ & 0.501 & & \\
\hline & EBRT (yes vs. no) & $1.09(0.57-2.07)$ & 0.791 & & \\
\hline & Brachytherapy (yes vs. no) & $0.74(0.25-2.20)$ & 0.590 & & \\
\hline & RP (yes vs. no) & $1.21(0.56-2.65)$ & 0.628 & & \\
\hline
\end{tabular}

Abbreviations: ADT, androgen deprivation therapy; BC, bladder cancer; PC, prostate cancer; CIS ,carcinoma in situ; BCG, Bacillus Calmette-Guérin; PSA, prostate-specific antigen; EBRT, external beam radiotherapy; RP, radical prostatectomy.

\section{Statistical analysis}

For the continuous variables, median and IQRs were reported. The categorical variables were summarized as frequencies and percentages. We evaluated the differences in the distribution of variables, using the chi-square test for categorical variables and a two-sample $t$-test or the Wilcoxon rank sum test for continuous variables, as appropriate. RFS defined as the time from the diagnosis of $\mathrm{BC}$ to the confirmation of disease recurrence or censoring was estimated by the Kaplan-Meier method and compared using the log-rank test. To examine the relationship between the time and mean number of recurrences, we calculated the mean cumulative function using ADT as the time-dependent covariate [31]. We estimated HRs and corresponding $95 \%$ CIs for the time to first and subsequent recurrences, using Univariable and multivariable Andersen and Gill models [32]. All statistical tests were two-tailed, and $P$-values $<0.05$ were considered significant. Statistical analyses were performed using SAS statistical software, version 9.2 (SAS Institute, Cary, NC).

\section{ACKNOWLEDGMENTS}

We thank the following doctors for their assistance in data collection: Drs. Shusei Fusayasu, Kohei Mori, Masahiro Inoue, Terukazu Hirasawa, Hiroaki Ishida, Nobuhiko Mizuno, Masahiro Yanagisawa, Koji Hoshino, Naoya Fujikawa, Kentaro Muraoka, Akitoshi Takizawa, Tatsuaki Onuki, Takehiro Tajiri, and Naoki Sakai. We also thank Prof. Takehiko Ogawa for preparing the manuscript.

\section{Disclosure of potential conflicts of interest}

No potential conflicts of interest were disclosed.

\section{Authors' contributions}

Conception and design: K. Izumi, H. Miyamoto, H. Uemura

Development of methodology: K. Izumi, M. Taguri 
Acquisition of data (provided animals, acquired and managed patients, provided facilities, etc.): Y. Hara, T. Kishida, K. Chiba, T. Murai, K. Hirai, K. Suzuki, K. Fujinami, T. Ueki, K. Udagawa, K. Kitami, M. Moriyama, Y. Miyoshi, F. Tsuchiya, I. Ikeda, K. Kobayashi, K. Noguchi Analysis and interpretation of data (e.g., statistical analysis, biostatistics, computational analysis): M. Taguri, M. Sato, S. Morita

Writing, review, and/or revision of the manuscript: K. Izumi, M. Taguri, H. Miyamoto, H. Uemura

Administrative, technical, or material support (i.e., reporting or organizing data, constructing databases): S. Morita

Study supervision: $\mathrm{H}$. Uemura

\section{GRANT SUPPORT}

This study was supported by a Grant-in-Aidfor Scientific Research (C) from the Ministryof Education, Culture, Science and Technology.

\section{REFERENCES}

1. Siegel R, Naishadham D, Jemal A. Cancer statistics. CA Cancer J Clin. 2013; 63:11-30.

2. Jemal A, Bray F, Center MM, Ferlay J, Ward E, Forman D. Global cancer statistics. CA Cancer J Clin. 2011; 61:69-90.

3. Shang PF, Kwong J, Wang ZP, Tian J, Jiang L, Yang K, Yue ZJ, Tian JQ. Intravesical Bacillus Calmette-Guérin versus epirubicin for Ta and T1 bladder cancer. Cochrane database Syst Rev. 2011; CD006885.

4. Sievert KD, Amend B, Nagele U, Schilling D, Bedke J, Horstmann M, Hennenlotter J, Kruck S, Stenzl A. Economic aspects of bladder cancer: what are the benefits and costs? World J Urol. 2009; 27:295-300.

5. Hartge P, Harvey EB, Linehan WM, Silverman DT, Sullivan JW, Hoover RN, Fraumeni JF. Unexplained excess risk of bladder cancer in men. J Natl Cancer Inst. 1990; 82:1636-40

6. Freedman ND, Silverman DT, Hollenbeck AR, Schatzkin A, Abnet CC. Association between smoking and risk of bladder cancer among men and women. JAMA. 2011; 306:737-45.

7. Miyamoto H, Yang Z, Chen $Y-T$, Ishiguro H, Uemura H, Kubota Y, Nagashima Y, Chang Y-J, Hu Y-C, Tsai M-Y, Yeh S, Messing EM, Chang C. Promotion of bladder cancer development and progression by androgen receptor signals. J Natl Cancer Inst. 2007; 99:558-68.

8. Hsu J-W, Hsu I, Xu D, Miyamoto H, Liang L, Wu X-R, Shyr C-R, Chang C. Decreased tumorigenesis and mortality from bladder cancer in mice lacking urothelial androgen receptor. Am J Pathol. 2013; 182:1811-20.
9. Li Y, Izumi K, Miyamoto H. The role of the androgen receptor in the development and progression of bladder cancer. Jpn J Clin Oncol. 2012; 42:569-77.

10. Miyamoto H, Yao JL, Chaux A, Zheng Y, Hsu I, Izumi K, Chang C, Messing EM, Netto GJ, Yeh S. Expression of androgen and oestrogen receptors and its prognostic significance in urothelial neoplasm of the urinary bladder. BJU Int. 2012; 109:1716-26.

11. Mir C, Shariat SF, van der Kwast TH, Ashfaq R, Lotan Y, Evans A, Skeldon S, Hanna S, Vajpeyi R, Kuk C, Alkhateeb S, Morote J, van Rhijn BWG, et al. Loss of androgen receptor expression is not associated with pathological stage, grade, gender or outcome in bladder cancer: a large multi-institutional study. BJU Int. 2011; 108:24-30.

12. Zheng $\mathrm{Y}$, Izumi $\mathrm{K}$, Yao JL, Miyamoto $\mathrm{H}$. Dihydrotestosterone upregulates the expression of epidermal growth factor receptor and ERBB2 in androgen receptor-positive bladder cancer cells. Endocr Relat Cancer. 2011; 18:451-64.

13. Boorjian SA, Heemers HV, Frank I, Farmer SA, Schmidt LJ, Sebo TJ, Tindall DJ. Expression and significance of androgen receptor coactivators in urothelial carcinoma of the bladder. Endocr Relat Cancer. 2009; $16: 123-37$.

14. Singer EA, Golijanin DJ, Miyamoto H, Messing EM. Androgen deprivation therapy for prostate cancer. Expert Opin Pharmacother. 2008; 9:211-28.

15. Akaza H, Usami M, Hinotsu S, Ogawa O, Kagawa S, Kitamura T, Tsukamoto T, Naito S, Hirao Y, Murai M, Yamanaka H. Characteristics of patients with prostate cancer who have initially been treated by hormone therapy in Japan: J-CaP surveillance. Jpn J Clin Oncol. 2004; 34:329-36.

16. Kinoshita Y, Singh A, Rovito PM, Wang CY, Haas GP. Double primary cancers of the prostate and bladder: a literature review. Clin Prostate Cancer. 2004; 3:83-6.

17. Anderson JR, Cain KC, Gelber RD. Analysis of survival by tumor response. J Clin Oncol. 1983; 1:710-9.

18. Gakis G, Stenzl A. Gender-specific differences in muscleinvasive bladder cancer: the concept of sex steroid sensitivity. World J Urol. 2013; 31:1059-64.

19. Boorjian S, Cowan JE, Konety BR, DuChane J, Tewari A, Carroll PR, Kane CJ. Bladder cancer incidence and risk factors in men with prostate cancer: results from Cancer of the Prostate Strategic Urologic Research Endeavor. J Urol. 2007; 177:883-7; discussion 887-8.

20. Hsu I, Chuang K-L, Slavin S, Da J, Lim W-X, Pang S-T, O'Brien JH, Yeh S. Suppression of ER $\beta$ signaling via ER $\beta$ knockout or antagonist protects against bladder cancer development. Carcinogenesis. 2014; 35:651-61.

21. Hsu I, Yeh C, Slavin S, Miyamoto H, Netto GJ, Tsai Y, Muyan M, Wu X, Messing EM, Guancial EA, Yeh S. Estrogen receptor alpha prevents bladder cancer via 
INPP4B inhibited akt pathway in vitro and in vivo. Oncotarget. 2014; 5:7917-35.

22. Izumi $\mathrm{K}$, Zheng $\mathrm{Y}$, Li $\mathrm{Y}$, Zaengle J, Miyamoto $\mathrm{H}$. Epidermal growth factor induces bladder cancer cell proliferation through activation of the androgen receptor. Int J Oncol. 2012; 41:1587-92.

23. Hsieh T-F, Chen C-C, Ma W-L, Chuang W-M, Hung X- F, Tsai Y-R, Lin M-HA, Zhang Q, Zhang C, Chang C, Shyr $\mathrm{C}-\mathrm{R}$. Epidermal growth factor enhances androgen receptormediated bladder cancer progression and invasion via potentiation of AR transactivation. Oncol Rep. 2013; 30:2917-22.

24. Li Y, Zheng Y, Izumi $\mathrm{K}$, Ishiguro $\mathrm{H}$, Ye $\mathrm{B}$, Li F, Miyamoto $H$. Androgen activates $\beta$-catenin signaling in bladder cancer cells. Endocr Relat Cancer. 2013; 20:293-304.

25. Lin C, Yin Y, Stemler K, Humphrey P, Kibel AS, Mysorekar IU, Ma L. Constitutive $\beta$-catenin activation induces male-specific tumorigenesis in the bladder urothelium. Cancer Res. 2013; 73:5914-25.

26. Izumi K, Zheng Y, Hsu J, Chang C, Miyamoto H. Androgen receptor signals regulate UDP-glucuronosyltransferases in the urinary bladder: A potential mechanism of androgeninduced bladder carcinogenesis. Mol Carcinog. 2013; 52:94-102.

27. Izumi $\mathrm{K}$, Li Y, Ishiguro H, Zheng $\mathrm{Y}$, Yao JL, Netto GJ, Miyamoto H. Expression of UDP-glucuronosyltransferase
1A in bladder cancer: Association with prognosis and regulation by estrogen. Mol Carcinog. 2014; 53:314-24.

28. Overdevest JB, Knubel KH, Duex JE, Thomas S, Nitz MD, Harding MA, Smith SC, Frierson HF, Conaway M, Theodorescu D. CD24 expression is important in male urothelial tumorigenesis and metastasis in mice and is androgen regulated. Proc Natl Acad Sci U S A. 2012; 109:E3588-96.

29. Sylvester RJ, van der Meijden APM, Oosterlinck W, Witjes JA, Bouffioux C, Denis L, Newling DWW, Kurth K. Predicting recurrence and progression in individual patients with stage Ta T1 bladder cancer using EORTC risk tables: a combined analysis of 2596 patients from seven EORTC trials. Eur Urol. 2006; 49:466-5; discussion 475-7.

30. Miyamoto H, Miller JS, Fajardo DA, Lee TK, Netto GJ, Epstein JI. Non-invasive papillary urothelial neoplasms: the 2004 WHO/ISUP classification system. Pathol Int. 2010; 60:1-8.

31. Nelson WB. Recurrent Events Data Analysis for Product Repairs, Disease Recurrences, and Other Applications Philadelphia, PA: SIAM; 2003.

32. Andersen PK, Gill RD. Cox's Regression Model for Counting Processes: A Large Sample Study. Ann Stat. 1982; 10:1100-20. 